EPJ Web of Conferences 62, 05006 (2013)

DOI: $10.1051 /$ epjconf/20136205006

(C) Owned by the authors, published by EDP Sciences, 2013

\title{
LICORNE: A new and unique facility for producing intense, kinematically focused neutron beams at the IPN Orsay
}

\author{
J.N. Wilson ${ }^{1, a}$, M. Lebois ${ }^{1}$, P. Halipre ${ }^{1}$, B. Leniau ${ }^{1}$, I. Matea $^{1}$, D. Verney ${ }^{1}$, S. Oberstedt ${ }^{2}$, \\ R. Billnert ${ }^{2,3}$, A. Oberstedt ${ }^{3}$, G. Georgiev ${ }^{4}$, and J. Ljungvall ${ }^{4}$ \\ ${ }^{1}$ Institut de Physique Nucléaire Orsay, 91406 Orsay, France \\ ${ }^{2}$ European Commission, DG Joint Research Centre IRMM, Retieseweg 111, 2440 Geel, \\ Belgium \\ ${ }^{3}$ Fundamental Fysik, Chalmers Tekniska Högskola, 41296 Göteborg, Sweden \\ ${ }^{4}$ CSNSM Orsay, 91405 Orsay, France
}

\begin{abstract}
LICORNE is a new neutron source recently installed at the tandem accelerator of the Institut de Physique Nucléaire d'Orsay, where a Li7-beam is used to bombard a hydrogen-containing target to produce an intense forward-directed neutron beam. The directionality of the beam, which is the unique characteristic of LICORNE, will permit the installation of $\gamma$-ray detectors dedicated to the investigation of fission fragment de-excitation which are unimpeded by neutrons from the source. A first experimental program will focus on the measurement of prompt $\gamma$-ray emission in the neutron-induced fission of fertile and fissile isotopes at incident neutron energies relevant for the core design of Generation-IV nuclear reactors. Other potential uses of the LICORNE facility for both fundamental and applied physics research are also presented.
\end{abstract}

\section{Introduction}

Conventional quasi-mono-energetic neutron sources produce neutrons isotropically via direct reactions on light nuclei (e.g. d(d,p)n or $\left.{ }^{7} \mathrm{Li}(\mathrm{p}, \mathrm{n})^{7} \mathrm{Be}\right)$.

The lack of directionality means that typically less than 1 percent of the source neutrons produced can be used for irradiating samples, the vast majority instead contributing to the room background. However, natural collimation of neutron beams can be achieved if the neutrons are produced using a reaction in inverse kinematics where the projectile is much heavier than the target. Neutron production via this method thus combines the best features of white neutron sources (collimated beams) and conventional quasi-mono-energetic neutron sources (high neutron fluxes at short distances).

The kinematic focusing technique clearly offers some distinct advantages over standard isotropic quasi-mono-energetic sources:

1. The focusing enhances the available neutron flux by a factor of between 25 and 100 .

2. The lack of neutron emission at most angles results in much lower fast and thermal scattered neutron backgrounds in the experimental hall.

\footnotetext{
ae-mail: wilson@ipno.in2p3.fr
}

This is an Open Access article distributed under the terms of the Creative Commons Attribution License 2.0, which permits unrestricted use, distribution, and reproduction in any medium, provided the original work is properly cited. 


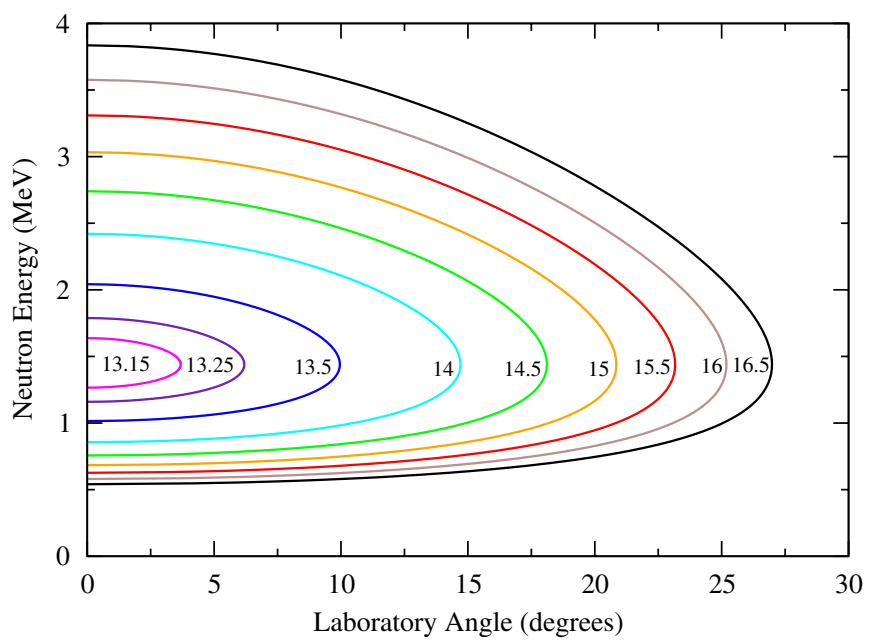

Figure 1. Kinematic curves relating the angle of neutron emission to neutron energy in the laboratory frame for different ${ }^{7} \mathrm{Li}$ bombarding energies from $13.15-16.5 \mathrm{MeV}$, calculated using two-body relativistic kinematics.

3. The placement of sensitive detectors adjacent to the neutron source becomes feasible, without the necessity for heavy shielding.

4. The neutrons produced lie in the energy range $0.5-4 \mathrm{MeV}$, exactly in the region of interest for generation IV fast reactor concepts.

The LICORNE neutron source [1] is initially intended for performing fundamental studies of the nuclear fission process and associated nuclear data measurements related to 4th generation nuclear reactors. A first experimental program will involve the study of prompt gamma-ray emission in fission since the directional neutron beam will allow placement of gamma-ray detectors out-of-flux but adjacent to the sample to be irradiated. However, other potential uses of LICORNE span several different research fields and include gamma-spectroscopy of neutron-induced reactions, measurements of capture and inelastic scattering cross sections, non-destructive assay of nuclear waste, activation measurements and irradiation for the aerospace industry.

\section{Inverse kinematics}

The main advantage of inverse kinematics is the natural forward collimation of the reaction ejectiles. The heavier the projectile nucleus, the greater the collimation. For reactions which eject neutrons this will induce large enhancements of neutron fluxes at 0 degrees in the laboratory frame. The $\mathrm{p}\left({ }^{7} \mathrm{Li}, \mathrm{n}\right){ }^{7} \mathrm{Be}$ reaction is one of the most commonly used in direct kinematics to produce mono energetic neutrons, especially below $0.7 \mathrm{MeV}$ [2]. However, in inverse kinematics with a Li-beam, mono-energetic neutrons of $1.44 \mathrm{MeV}$ are produced at bombarding energies at the reaction threshold of $13.09 \mathrm{MeV}$. The results of 2-body relativistic kinematics calculations are shown on Figure 1. The kinematic curves for a given bombarding energy have two distinct peaks in the laboratory frame corresponding to forward emission and backward emission of neutrons in the centre of mass frame. The relative size of the principal (high energy) peak and satellite (low energy) peak is governed by the relativistic kinematics of the focusing and the angular distribution of emission in the centre of mass frame, which changes quite dramatically as a function of ${ }^{7} \mathrm{Li}$ bombarding energy.

The gain from the focusing and natural collimation can be expressed in terms of neutron flux enhancement over the non-inverse reaction. Near the threshold the enhancement factor is maximal 


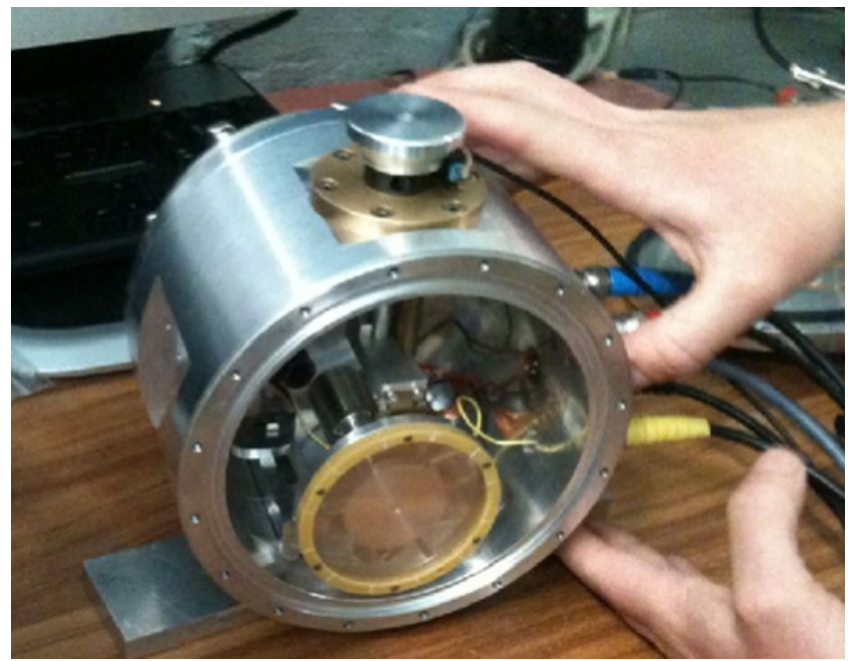

Figure 2. Photograph of the LICORNE neutron convertor chamber with the front face removed. The stack of rotating polypropylene targets can be seen.

(>100) since the emitted neutrons move with the centre of mass of the system which follows the ${ }^{7} \mathrm{Li}$ beam direction. As a consequence, close to the threshold energy, it is possible to produce very narrow ( $<5$ degrees) cones of neutrons. With increasing ${ }^{7} \mathrm{Li}$ bombarding energy, the cone broadens and the number of neutrons in a given solid angle decreases so the enhancement factor drops to around 20 at $16.5 \mathrm{MeV}$.

However, the huge gain in intensity due to the kinematic focusing is offset by corresponding losses from two other factors. Firstly, the available beam current of ${ }^{7} \mathrm{Li}$ is much lower than that available for protons in the non-inverse reaction because of the relative difficulty of extraction of ${ }^{7} \mathrm{Li}$-ions from the ion source. Secondly, the energy loss of ${ }^{7} \mathrm{Li}$ across a given target will be higher than that for protons due to its higher atomic number.

The current sputter source of the IPN tandem can produce ${ }^{7} \mathrm{Li}$ beam currents of up to $0.2 \mu \mathrm{A}$, but currents greater than $0.5 \mu \mathrm{A}$ may be achievable with the source upgrade which will take place soon. The maximum available fluxes from LICORNE are therefore expected to around $10^{7}$ neutrons/second/steradian for a thin polypropylene target $(4 \mu \mathrm{m})$ and $7 \times 10^{7}$ neutrons/second/ steradian for a thick $(28 \mu \mathrm{m})$ polypropylene target. These fluxes are comparable with other acceleratorbased neutrons sources, but LICORNE has the added advantage of a natural directionality and a much lower background.

\section{LICORNE design}

The LICORNE neutron converter sits in an aluminium chamber of diameter approximately $17 \mathrm{~cm}$. It is designed with a rotating polypropylene target of $4 \mu \mathrm{m}$ thickness and a diameter of $8 \mathrm{~cm}$ (see Figs. 2 and 3 ). The rotation is necessary to increase the irradiated surface area by a factor of 25 with respect to a fixed target. Polypropylene is not very resistant to radiation damage, and therefore the rotating target prolongs the lifetime of the target to around one week under normal experimental conditions.

Between one and 10 polypropylene targets can be stacked on the target wheel. The arms are made of tungsten to prevent nuclear reactions with ${ }^{7} \mathrm{Li}$ occurring.

Beam current and beam time structure can be measured with the beam stop, which consists of a $50 \mu \mathrm{m}$ gold foil and is connected to a high bandwidth, low noise amplifier just outside the reaction 


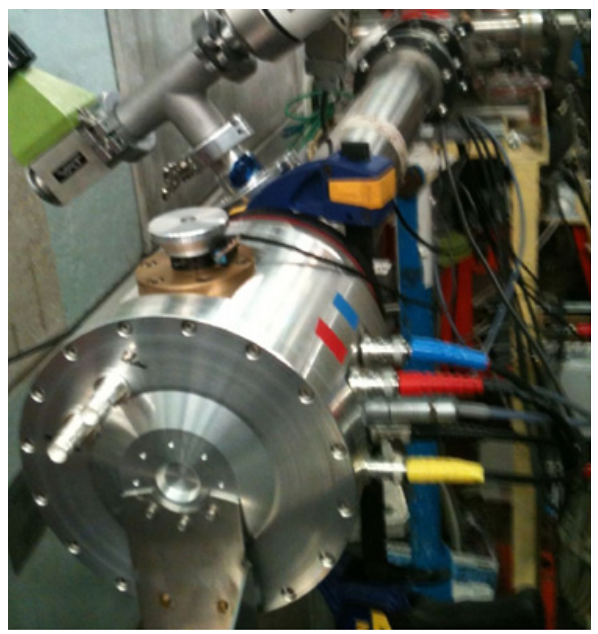

Figure 3. Photograph of the LICORNE chamber mounted at the end of the Orsay tandem accelerator beam-line.

chamber. An electric field of up to several hundred volts can be applied to a conducting ring around the gold foil to prevent loss of ejected electrons from the gold surface. The exit window of the LICORNE convertor front face is made of Aluminium and is only $0.3 \mathrm{~mm}$ thick. A mini camera, illuminating LED are included for beam-tuning and inspecting the targets from inside without having to break the vacuum of $<10^{-5}$ atmospheres. The beam spot is tuned by placing a retractable phosphorescent target in the path of the beam.

\section{Available fluxes}

A Monte-Carlo code has been developed for the purpose of predicting neutron spectra as a function of ${ }^{7} \mathrm{Li}$ bombarding energy at any point in space. The Monte-Carlo code was validated by simulating the detected spectra in EDEN [4], a liquid scintillator detector placed at different angles with respect to the beam axis. The neutron angular distribution and spectral shapes are well reproduced.

Once validated, the Monte-Carlo code was then used as a tool to simulate and understand the fluxes and neutrons spectra that would be available for a dedicated neutron source using the $\mathrm{p}\left({ }^{7} \mathrm{Li},{ }^{7} \mathrm{Be}\right) \mathrm{n}$ inverse reaction. Irradiation of extended samples at short distances is of particular interest. From the kinematic curves in Figure 1, it can be seen that for samples which cover up to 10 degrees in the laboratory, the energies of principal and satellite peaks will remain fairly constant. Figure 4 shows the corresponding neutron spectra for thin $(4 \mu \mathrm{m})$ and thick $(28 \mu \mathrm{m})$ polypropylene targets.

A thick polypropylene target can be used as a white neutron source producing a broad neutron spectrum between 0.5 and $4 \mathrm{MeV}$. For a $28 \mu \mathrm{m}$ thick target the energy of ${ }^{7} \mathrm{Li}$ ions drops from $16.5 \mathrm{MeV}$ to $13 \mathrm{MeV}$ during their traversal. The advantage is that the reaction rate is increased and the total flux available is 7 times higher (approx. $7 \times 10^{7} \mathrm{n} / \mathrm{s} /$ steradian). If the ${ }^{7} \mathrm{Li}$ beam is pulsed, then timeof-flight could be used at short distances to determine the neutron energy. However, the maximum beam current available in pulsed mode drops by a factor of 4 . The energy resolution of such time-offlight measurements would be dominated by the contribution of the $1.5 \mathrm{~ns}$ beam pulse width, so up to 10 energy bins for a flight distance of $20 \mathrm{~cm}$ should be possible.

\section{Coupling of LICORNE with the ORGAM spectrometer}

Since LICORNE produces neutron cones with opening angles of between 5 and 25 degrees an entire new class of experiments is now possible thanks to the possibility to place gamma detectors around 


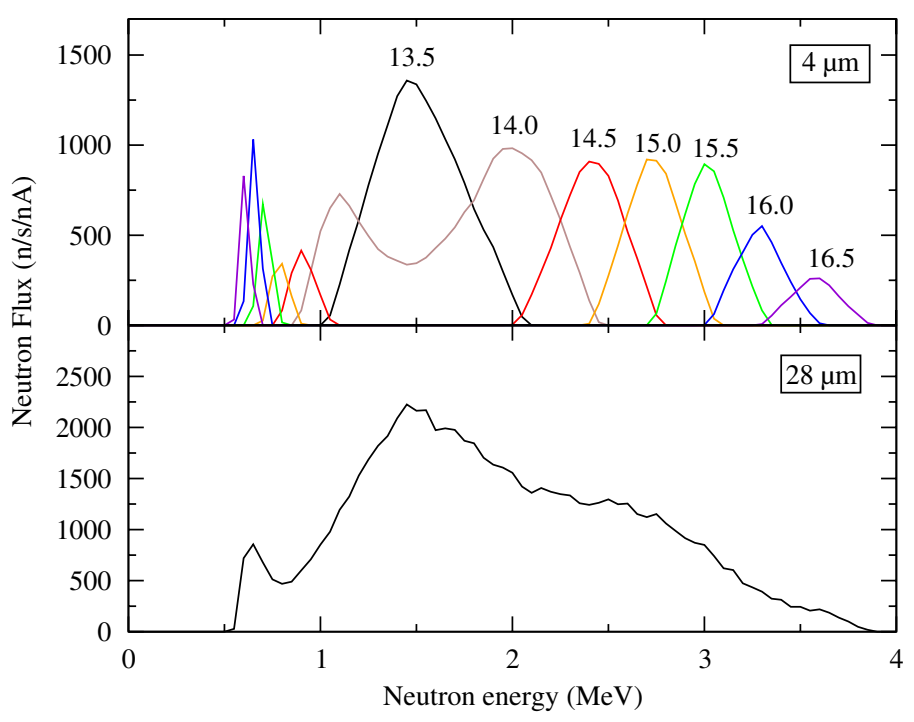

Figure 4. Neutron spectra for irradiation of extended samples covering 10 degrees at short distances (typically $20 \mathrm{~cm}$ ) from the LICORNE source for thin and thick polypropylene targets. The different coloured spectra correspond to seven different ${ }^{7} \mathrm{Li}$ bombarding energies between 13.5 and $16.5 \mathrm{MeV}$ which are shown above each peak.

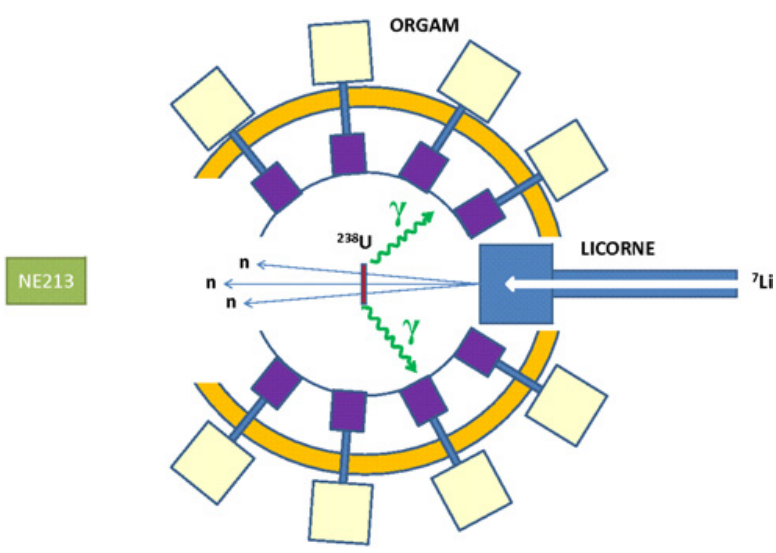

Figure 5. Schematic diagram of the coupling of LICORNE and the ORGAM spectrometer at IPN Orsay.

the sample to be irradiated, but outside the neutron cone. It will be possible to perform high-resolution spectroscopy of neutron-induced reactions. In 2014, the first coupling of LICORNE and the ORGAM high resolution Germanium array will take place (see Fig. 5). At least two types of experiment are possible:

(i) Spectroscopy of fast-neutron induced fission will allow the study of exotic fission fragments from fertile isotopes such as ${ }^{238} \mathrm{U}$ and ${ }^{232} \mathrm{Th}$. Since pulsed neutron beams are possible and Orgam is a triggerless system, fragments and their partners can be cleanly identified by the time-correlations present in decay of isomeric states. A first experiment in early 2014 will be performed to study production of exotic nuclei from fission of a sample of around $200 \mathrm{~g}$ of ${ }^{238} \mathrm{U}$. This should give a counting rate in the Orgam Ge detectors of several kHz. 
(ii) Spectroscopy of fast-neutron induced capture reactions using the two-step cascade method will obtain information on nuclear level densities above the neutron binding energy. This method relies on finding pairs of gamma-rays which sum to the energy of the compound nucleus formed. This method could be used with LICORNE and ORGAM and applied to fast-neutron capture at one or more different neutron energies.

\section{Conclusion}

LICORNE is a dedicated facility to produce intense, naturally collimated, quasi-mono-energetic neutron beams at the IPN Orsay. The kinematic focusing of the neutron allows gamma detectors to be placed near the irradiated sample and opens up a whole host of new possibilities for the study of neutron-induced reactions, in particular nuclear fission. The study of gamma-rays emitted in fission is for both fundamental and applied physics purposes. Fission gamma rays are important for the gamma-heating problem in innovative nuclear reactors. Fission gamma rays will also allow the tagging and study of exotic nuclei far from stability which are the precursors of the stable nuclei formed in supernova explosions. To sumarise, LICORNE is operational since June 2013 and offers some interesting and exciting new physics possibilities.

\section{References}

[1] M. Lebois, J.N. Wilson et al., Development of a kinematically focused neutron source with the p(7Li,n)7Be inverse reaction, Nuclear Instrumentation and Methods, A735 145 (2014)

[2] M. Chadwick, et al., ENDF/B-VII.1 nuclear data for science and technology: Cross sections, covariances, fission product yields and decay data, Nucl. Data Sheets 112 (12) (2011)

[3] S. Elbakr, et al., Measurements of neutron angular distributions from the $7 \mathrm{Li}(\mathrm{p}, \mathrm{n}) 7 \mathrm{Be}$ reaction, Nucl. Instr. and Meth. 105519 (1972)

[4] H. Laurent, et al., Eden: a neutron time-of-flight multidetector for decay studies of giant states, Nucl. Instr. and Meth. A 326517 (1993) 